\title{
Liberale Ordnungspolitik im demokratischen Diskurs
}

\section{GERHARD WEGNER ${ }^{* *}$}

\section{Liberal rule oriented market policy in democracy}

The article interrogates the legitimacy of rule-oriented, pro market policy recommendations ("ordnungspolitike") in democracy. Whereas classical policy recommendations in the tradition of Walter Eucken presume that welfare gains following from rule oriented policies guarantee for its legitimacy, democratic economy policy must rest on societal consent. It is shown that beliefs of citizens concerning the working conditions of a market order as well as political preferences will modify rules which economists identify for its best possible impact on welfare. However, as long as democracy remains stable, anti-market economic policies are unlikely to sustain in democracy.

Keywords: Liberalism, constitutional economics, institutional economics, democracy

Die Erschütterungen der internationalen Finanzmarktkrise im Jahre 2008 und die sich daran anschließende Krise im Euro-Währungsraum haben vordergründig einen neuen Konsens über die Notwendigkeit einer Ordnungspolitik begründet. Der in Profession und Feuilleton allenthalben zu hörende Ruf, dass „,eine Marktwirtschaft Regeln braucht", trifft gegenwärtig auf breite Resonanz. Dabei verbinden sich jedoch traditionale anti-marktwirtschaftliche Einstellungen, die gegen marktwirtschaftliche Entwicklungsdynamik per se Misstrauen hegen und deren generelle Eindämmung fordern, mit pro-marktwirtschaftlichen Überzeugungen, bei denen es um eine Funktionsverbesserung der Marktwirtschaft geht, um künftigen Finanzmarkt- und Währungskrisen vorzubeugen. Aus beiden Überzeugungen heraus lassen sich ordnungspolitische Regeln fordern, aus denen freilich ein unterschiedlicher Regelungsgehalt folgt. So haben Kommentatoren seriöser Zeitungen in Deutschland den Ausbruch der Finanzmarktkrise zum Anlass genommen, vor der Privatisierung der kommunalen Müllabfuhr zu warnen oder den allgemeinen gesetzlichen Mindestlohn zu fordern. Auch solche Forderungen firmieren derzeit als „Ordnungspolitik“, wohinter sich aber ein politisches Programm zur Überwindung des als monolithisch perzipierten „Neoliberalismus“ verbirgt. Umgekehrt streiten Ökonomen vor allem darum, welche Regulierungen in der Finanzbranche zieladäquat und umgehungsfest sein könnten und in welcher Weise staatliches Handeln an Regeln zu binden wäre, um eine Krisenverschärfung zu ver-

Beitrag eingereicht am 11.03.2011, nach doppelt verdecktem Gutachterverfahren überarbeitete Fassung angenommen am 15.07.2011.

** Prof. Dr. Gerhard Wegner, Lehrstuhl für Institutionenökonomie und Wirtschaftspolitik, Universität Erfurt, Nordhäuserstr. 63, 99089 Erfurt, Tel: +49 (0)361-737-4541, Fax: +49 (0)361737-4549, E-Mail: gerhard-wegner@uni-erfurt.de, Forschungsschwerpunkte: Ordnungstheorie und -politik, Theorie der Wirtschaftspolitik, Politischer und ökonomischer Liberalismus, Neue Institutionenökonomik, Systemwettbewerb in der EU. 
meiden. Die Brücke zwischen anti-marktwirtschaftlichen und pro-marktwirtschaftlichen Überzeugungen bildet dabei, und dies macht die Unübersichtlichkeit perfekt, ein von beiden Seiten erhobener wirtschaftsethischer Anspruch. Die mögliche Funktion von ordnungspolitischen Regeln und ihr Gehalt bleiben damit bis aufs Weitere unbestimmt. Der derzeitige gesellschaftliche Konsens über die Notwendigkeit von Regeln für die Märkte besteht darum nur vordergründig und überdeckt eine tiefe Kluft, die rasch sichtbar wird, sobald die gesetzgeberische Etablierung konkret wird. Im Folgenden soll das Konfliktfeld, das eine demokratische Ordnungspolitik charakterisiert, näher beschrieben werden. Denn demokratische Ordnungspolitik muss als Resultante inkonsistenter und widersprüchlicher Erwartungen begriffen werden, die mit Ordnungsregeln verknüpft werden. Anders als der traditionellen Ordnungspolitik der Freiburger Schule, wie sie von Walter Eucken begründet wurde, geht es einer demokratischen Ordnungspolitik nicht vordringlich und exklusiv um die Implementierung liberaler Regeln für eine funktionsfähige Marktwirtschaft. Sie bezieht ihre normativen Erwartungen über marktwirtschaftliche Ordnungsmuster, die mit Regeln hervorzubringen sind, vielmehr aus den kontingenten Ergebnissen öffentlicher Diskurse. Daraus ist wiederum nicht der Umkehrschluss zu ziehen, dass eine demokratische Ordnungspolitik auf die intendierte Überwindung oder nicht-intendierte Sklerosis (Olson) der marktwirtschaftlichen Ordnung angelegt wäre. Jedoch hat eine demokratische Ordnungspolitik andere Themenfelder zu bearbeiten, als sie sich für die traditionelle Ordnungspolitik gestellt haben:

- An die Stelle des substantiellen Liberalismus, der die traditionelle Ordnungspolitik Walter Euckens - ebenso wie den klassischen Liberalismus - kennzeichnet, tritt ein prozedural zu bestimmender Liberalismus, der seine Legitimation aus dem demokratischen Verfahren bezieht.

- Der Regelungsgehalt demokratischer Ordnungspolitik hängt von den kontingenten Präferenzen des Souveräns der Demokratie ab, welche die ökonomischen Gestaltungsabsichten bestimmen.

- Da demokratische Ordnungspolitik zwischen dem politisch Gewollten und marktwirtschaftlichen Funktionsprinzipien vermittelt, hängt ihre Realisierbarkeit zudem von epistemischen Bedingungen ab. Es ist nicht ausgemacht, ob der Souverän der Demokratie marktwirtschaftliche Funktionsprinzipien hinreichend berücksichtigt oder vielmehr nachholend - als Resultat unerwünschter Ordnungsfolgen - in politische Handlungsziele einpasst. Letzteres kann eine nur schwach präferierte, aber unvermeidlich erscheinende Liberalisierungspolitik bedeuten.

- Auch ordnungspolitische Regelbindung ist als ein experimenteller Prozess von Versuch und Irrtum zu konzipieren, was der Ungewissheit über die Folgen von Regeln geschuldet ist sowie Lernprozessen des Souveräns der Demokratie.

Auf diese Punkte wird im Folgenden thesenhaft eingegangen, um die aus der hier vertretenen Sicht zentralen Fragestellungen gegenwärtiger Ordnungspolitik zu umrei- 
Ben. Aus Gründen der gebotenen Kürze müssen die nachfolgenden Ausführungen skizzenhaft bleiben. ${ }^{1}$

\section{Ordnungspolitik als Variante eines substantiellen ökonomischen Liberalismus}

Ordnungspolitik beabsichtigt die konstitutionelle Absicherung einer liberalen Marktverfassung, weil die marktwirtschaftliche Ordnung nur begrenzt als selbststabilisierend erachtet wird. Bei aller Unterschiedlichkeit zwischen den verschiedenen Positionen des ordnungsökonomischen Denkens, wie sie bei Vertretern wie Hayek, Eucken, Böhm, Rüstow oder Röpke zum Ausdruck kommt, zielt die klassische Ordnungspolitik auf die konstitutionelle Verankerung einer wettbewerblichen Marktordnung. Von der immer wiederkehrenden Distanznahme zum klassischen englischen Liberalismus darf man sich dabei nicht irritieren lassen, ebenso wenig von Begrifflichkeiten wie „Soziale Marktwirtschaft“ oder „Dritter Weg“. Auch wenn die deutschen Vertreter des Ordo-Liberalismus wie Eucken, Röpke oder Böhm ein ausgeprägtes Gespür für die soziale Frage des 19. und frühen 20. Jahrhunderts besaßen und sich hierin von Hayek unterschieden, so hatten sie mit ihrer Leitidee keineswegs ordnungspolitische Mischformen oder gar den Wohlfahrtsstaat der späteren Nachkriegszeit im Sinn. Die Attraktivität des Begriffes „Soziale Marktwirtschaft“, wie sie gegenwärtig in Transformations- und Schwellenländern registrierbar ist, verdankt sich wesentlich dem verbreiteten Missverständnis, dass es sich um einen „dritten Weg“ zwischen Marktwirtschaft und Sozialismus handeln würde. Zwar vermag dieses Missverständnis bis heute ein anhaltendes, auch internationales Interesse am Konzept der Sozialen Marktwirtschaft zu wecken. Dennoch bleibt festzuhalten, dass sämtliche Vertreter ordnungspolitischen Denkens eine liberale Wettbewerbsgesellschaft etablieren wollten und hierfür nach institutionellen Grundlagen suchten. Regulatorische Eingriffe und Beschränkungen des freien Wettbewerbs, aber auch sozialpolitische Korrekturen, waren dabei auf Ausnahmen zu begrenzen, auch wenn den Ordoliberalen bewusst war, dass erst die Überwindung von Armut für breite Schichten einer marktwirtschaftlichen Ordnung allgemeine Legitimation verschaffen könne. Eine Sozialpolitik, wie sie die meisten hoch entwickelten Volkswirtschaften betreiben, erhält jedoch deshalb geringen konzeptionellen Rang, weil sie im Sinne einer Wohlstandspolitik weder möglich noch nötig wäre: Denn nur eine stabile Wettbewerbsordnung wäre das einzige Mittel, den Wohlstand breiter gesellschaftlicher Schichten nachhaltig anzuheben. Diese empirisch durchaus valide Annahme macht sozialpolitische Eingriffe - etwa zum Zwecke des Gesundheitsschutzes von Arbeitern - lediglich in solchen Fällen flankierend erforderlich, in denen der Wettbewerb dauerhaft Funktionslücken aufweist. Dabei handelt es sich jedoch nicht um die Implementierung einer umfassenden Politik der Umverteilung, wie sie später auch von bekennenden Anhängern der Sozialen Marktwirtschaft mitgetragen oder gar betrieben wurd e.

Anders als das Rubrum „sozial“ vermuten lässt, basiert die traditionelle Ordnungspolitik trotz zahlreicher Adaptionen und Modifikationen im Wesentlichen darauf, das Smith'sche System der natürlichen Freiheit institutionell abzusichern, und zwar vor 
allem in solchen Fällen, in denen der Wettbewerb als Selbstregulativ unzureichend funktioniert oder von einer Aushöhlung durch das Monopolstreben der Marktteilnehmer bedroht ist. Dies impliziert eine konsequente Verwirklichung der materiellen Rechtsstaatlichkeit und die Etablierung einer Wirtschaftsordnung ohne Privilegien für Personen oder Gruppen, gleichviel ob es sich um steuerliche, transferbezogene oder Marktzugangsprivilegien handelt. Da Privilegien stets Wettbewerbsbeschränkungen bedeuten und damit die ökonomische Kontrollfunktion des Wettbewerbs für die Allokation von Ressourcen herabsetzen, muss Ordnungspolitik die Gleichheit der Teilnahmebedingungen aller Marktakteure am Wettbewerb sicherstellen. Insbesondere Hayek weist der materiellen Rechtsstaatlichkeit eine exklusive Stellung zu, weshalb er in besonderer Nähe zum klassischen ökonomischen Liberalismus steht. Dabei sind die wirtschaftspolitischen Unterschiede zu Eucken - entgegen einer verbreiteten Auffassung - gering. Sie finden sich vor allem in der Begründungsweise für eine Wettbewerbsordnung, die bei Eucken weniger deduktiv als bei Hayek ausgelegt ist. Des Weiteren in einer größeren Aufmerksamkeit, die Eucken abwägenden Erörterung wirtschaftspolitischer Handlungsalternativen schenkt. Sämtliche Vertreter der traditionellen Ordnungsökonomik in der Nachfolge Euckens argumentieren jedoch im Sinne eines ökonomischen Liberalismus, den ich als substantiellen ökonomischen Liberalismus bezeichnen möchte. ${ }^{2}$ Dieser gewinnt seine politischen Normen nicht aus einem Verfahren der Zustimmung, sondern aus einer ökonomischen Theorie über die Bedingung der Entstehung von Wohlfahrt. In der Lehrbuchvariante läuft dies auf die Empfehlung hinaus, nur im Falle von Marktversagen wirtschaftspolitische Korrekturen vorzunehmen, ansonsten den Wettbewerb wirken zu lassen. Darüber hinausgehende Eingriffe gefährden die Wettbewerbsordnung und sind darum zu unterbinden. Dabei spielt es keine Rolle, wie die Bürger von ihrem aktuellen Erfahrungs- oder Bildungsstand eine solche Politik beurteilen würden.

Substantiell ist diese Variante des ökonomischen Liberalismus auch deswegen zu nennen, weil sie der Verwendung des Marktversagensbegriffes enge Grenzen setzt. Der zurückhaltende Gebrauch findet Unterstützung in der Public-Choice-Theorie, die zwischen hinnehmbaren Marktunvollkommenheiten und Marktversagen unterscheidet, auch wenn es hierbei zu theoretischen Unterscheidungsproblemen kommen kann. Eine liberale Interpretation des Begriffs „Marktversagen“ unterzieht Funktionslücken auf Märkten mehrschrittigen Prüfungen, bevor eine wirtschaftspolitische Handlungsempfehlung hinreichend begründet erscheint. Dabei ist insbesondere dem wirtschaftspolitischen Lenkungswissen und der absehbaren Vereinnahmung durch Gruppeninteressen auf Seiten der wirtschaftspolitischen Akteure Aufmerksamkeit zu schenken, damit nicht jeder Kandidat einer Marktunvollkommenheit zum Vorwand für ein Primat der Politik über die Ökonomie genommen werden kann. In dieser Hinsicht erweist sich die Theorie des Wohlfahrtsoptimums und die Theorie des Marktversagens als ihrem Korrelat als defizitär. Denn allokationsverbessernde Korrekturmög-

2 Wegner (2009); zur traditionellen Ordnungsökonomik gehören ausdrücklich nicht vertragstheoretisch argumentierende Vertreter des Liberalismus, die (wie etwa Vanberg) die Freiheit der Märkte aus markttheoretischen Gründen gutheißen, ohne damit einer Entscheidung des Souveräns der Demokratie vorgreifen zu wollen. Hier bildet allein die Zustimmung der Bürger das unhintergehbare Legitimationskriterium. 
lichkeiten zur Behebung von Marktversagen lassen sich beliebig ausdenken, können aber auch die Wettbewerbsdynamik beschränken. Darum könnten sich solche Eingriffe auf lange Sicht als dysfunktional für die marktwirtschaftliche Ordnung erweisen. ${ }^{3}$

Die traditionelle Ordnungsökonomik stellt eine Variante des substantiellen ökonomischen Liberalismus dar und schöpft ihre Legitimation aus einer ökonomischen Theorie über die Entstehung von Wohlstand. Es handelt sich um ein theoriegestütztes Leitbild, das Habermas als „expertokratisch“ bezeichnen würde oder Rawls als „vernünftige umfassende Lehre" charakterisiert hätte. ${ }^{4}$ Ohne den Gesetzgeber oder auch die Adressaten einer Wohlstandspolitik zu konsultieren, leitet der substantielle ökonomische Liberalismus ab, dass eine Wettbewerbsordnung auf einer privilegienfreien Wirtschaftspolitik basiert und entsprechender institutioneller Absicherungen bedarf. Auch wenn diese Wirtschaftspolitik eine sozialpolitische Korrektur von Bildungschancen nicht ausschließt und möglicherweise sogar erfordert, bildet der materielle Rechtsstaat den institutionellen Kern für eine wohlstandssichernde Wettbewerbsordnung. ${ }^{5}$ Der materielle Rechtsstaat erfährt allerdings eine enge Auslegung dadurch, als er demokratischen Gestaltungsversuchen der marktwirtschaftlichen Ordnung - Hayek würde sie als „konstruktivistisch“ bezeichnen - enge Grenzen setzt. Denn solche Gestaltungen lassen sich nur mit einem formalen Rechtsstaatsbegriff in Einklang bringen; materiell bedeuten sie stets die Begünstigung von Gruppen, auch wenn solche Politiken mit einem Gemeinwohlziel begründet werden.

Typischerweise entgehen die wettbewerbsverzerrenden Effekte einer gezielten Einflussnahme auf den Marktprozess zunächst der öffentlichen Aufmerksamkeit; gleichwohl können solche Prozesswirkungen die Funktionalität von Marktprozessen nachhaltig stören. Dies kann gegenwärtig exemplarisch im Falle der Förderung erneuerbarer Energien beobachtet werden, die sich anfangs eines breiten gesellschaftlichen Konsens erfreuen konnte, inzwischen aber zu einer kostspieligen und auch ökologisch dysfunktionalen Belastung für die Bürger ausgewachsen ist, deren Legitimationskrise möglicherweise erst noch bevorsteht. ${ }^{6}$ Es entspricht dem Selbstverständnis der Ordnungspolitik, langfristige Funktionsstörungen der Marktwirtschaft als Folge einer intervenierenden Wirtschaftspolitik „expertokratisch“ zu analysieren und im Zweifel gegen die öffentliche Meinung vorzutragen. Damit müssen nicht vorherrschende öffentliche Motive für politische Gestaltungsversuche in Abrede gestellt oder herabgewürdigt werden. Gerade Walter Eucken ist regulatorischen Motiven der Gesellschaftspolitik stets mit Respekt begegnet, wie seine umsichtige Behandlung der sozia-

3 Ein Beispiel hierfür bietet die großzügige Auslegung des Marktversagensbegriffs durch das deutsche GWB, das vermeintliche natürliche Monopole jahrzehntelang zu Lasten der Verbraucher und des Wettbewerbs geschützt hat.

$4 \quad$ Vgl. Rawls (1998) und Habermas (1992: 231 u. 530).

5 Die Unterschiede zwischen dem Begriff der Rechtsstaatlichkeit aus der deutschen Rechtslehre und der „Rule of Law“ seien hier vemachlässigt.

6 Vgl. hierzu die Kritik des wissenschaftlichen Beirats beim Bundeswirtschaftsministeriums gegen das Gesetz zur Förderung erneuerbarer Energien aus dem Jahre 2004 (www.stoerwind.de/ WissBeirat.pdf), in dem vor einer Kostenexplosion gewarnt wurde; der Beirat hat seine Kritik an der Vorgabe von Mengenzielen angesichts der neuesten Entwicklung in der Energiepolitik bekräftigt (www.bmwi.de/BMWi/Redaktion/PDF/P-R/pressezettel-erneuerbare-energie). 
len Frage bezeugt. Da aber politische Gestaltungsabsichten in ihrer gesetzgeberischen Umsetzung in der Regel Umverteilungen, Beschränkungen der Vertragsfreiheit einschließlich Preisregulierungen und Beschränkung der Eigentumsrechte bedeuten und damit ein Ordnungsrisiko in sich bergen, reicht es nicht hin, wenn sich eine Gesellschaft auf die Notwendigkeit einer Gestaltung des Marktgeschehens abstrakt verständigt. Die Ordnungsfolgen der Gesetzgebung sind stets einzubeziehen, was die Möglichkeit einschließt, von wirtschaftspolitischen Eingriffen im Zweifel abzusehen. Aus Sicht der Ordnungsökonomik können nur starke Gründe zugunsten eines Gestaltungsinteresses wirtschaftspolitische Handlungen rechtfertigen, die den Wet tbewerb einer Beschränkung oder Verzerrung unterwerfen.

Im ordnungsökonomischen Denken, so lässt sich resümieren, stellt der dynamische Wettbewerb selbst ein Gemeinwohlziel dar.7 Wenn Politik oder Rechtsprechung andere Ziele mit Gemeinwohlanspruch definieren, so können diese keinen automatischen Vorrang beanspruchen, sobald dadurch Wettbewerb verdrängt würde. Es handelt sich vielmehr um einen Abwägungskonflikt, der mit Hilfe ordnungspolitischer Expertise aufzulösen und zu entscheiden wäre. Die demokratische Öffentlichkeit oder politische Entscheidungsprozesse können im ordnungspolitischen Denken keine Prärogative beanspruchen, ebensowenig wie dies im Falle eines Konflikts zwischen wirtschaftspolitischen Handlungen und den Grundrechten der Fall wäre. Der ökonomischen Freiheit kommt selbst grundrechtlicher Status zu, zumal diese nach vorherrschender Meinung als Ausfluss der Grundrechte gedeutet werden kann.

\section{Das Legitimationsproblem marktwirtschaftlicher Ordnungspolitik in der Demokratie}

Es ist evident, dass in einer demokratischen Ordnung das ordnungsökonomische Legitimationsprinzip, welches sich expertokratisch auf die institutionelle Bedingung für gesellschaftlichen Wohlstand beruft, nicht mehr hinreicht. In dem Maße, wie die Agenten des Souveräns der Demokratie öffentlicher Kontrolle ausgesetzt sind und die nicht zu leugnende Einflussnahme durch Gruppeninteressen zumindest eingehegt werden kann, entscheiden die Bürger über die geltenden wirtschaftspolitischen Prinzipien selbst. Dann aber können ordnungsökonomische Prinzipien mit den empirischen politischen Präferenzen der Bürger konfligieren. Verstöße gegen ordnungspolitische Prinzipien, welche für eine Marktwirtschaft konstitutiv sind, lassen sich in dieser Konstellation nur noch mit großen Einschränkungen als „Verschwörung“ politischer Akteure gegen den Souverän der Demokratie deuten. Zwar bestehen auch in Demokratien vielfältige Möglichkeiten, den Wählerwillen zu verfälschen und Sonderintere ssen zulasten eines Wählervotums zu bedienen. Auf Dauer jedoch können zentrale Leitbilder der demokratischen Wirtschaftspolitik - z. B. ein Sozialstaatsprinzip - nicht gegen den Souverän der Demokratie betrieben werden. Daraus erwachsen Folgen für die Bewertung einer Wirtschaftspolitik, die klassische ordnungspolitische Prinzipien verletzt. Zwar lässt sich diese Politik als ordnungsinkonform kritisieren, aber man

\footnotetext{
Dezidierte Vertreter dieser Position sind u.a. Eucken, Hoppmann, Streit oder Möschel, die immer wieder den Wettbewerb per se als Gemeinwohlziel aufgefasst und eine Instrumentalisierung des Wettbewerbs für politische Ziele kritisch betrachtet haben.
} 
kann ihr kaum ihre Legitimation absprechen, nur weil Bürgern der Sinn ordnungspolitischer Regeln nicht einleuchtet.

Um der ordnungspolitischen Kritik Nachdruck zu verleihen, haben Liberale unterschiedlicher Provenienz zumeist ein Negativszenario entworfen, das bei Nichtbeachtung ordnungspolitischer Prinzipien demokratischen Gesellschaften eine „institutionelle Sklerose“ (Olson) oder gar einen „Weg in die Knechtschaft" (Hayek) voraussagt. In einer umfassenden Theorie der Wirtschaftsgeschichte verwerfen North,Wallis und Weingast (2009) solche Negativszenarien für die demokratische Wirtschaftspolitik. Gleichfalls korrigieren sie die in der Public-Choice-Theorie lange Zeit vorherrschende Auffassung, wonach demokratische Wirtschaftspolitik auf eine immer umfangreichere Privilegienwirtschaft hinausliefe, die den Wohlstand dauerhaft aufs Spiel setzen würde. Die Referenz für eine solche modifizierende Betrachtungsweise bieten vordemokratische und autokratische Wirtschaftsordnungen, wie sie einen großen Teil der Wirtschaftsgeschichte beherrschen und auch heute noch in der Weltwirtschaft überwiegen. Die Autoren treten der Auffassung entgegen, dass Demokratien auf Sklerose der marktwirtschaftlichen Ordnung angelegt wären, auch wenn sie bekanntermaßen kein Ideal einer liberalen Wirtschaftspolitik verwirklichen. ${ }^{8} \mathrm{Im}$ Vergleich zu vordemokratischen „Limited Access Orders“ unterliegt eine gezielte, auf Verletzung von Rechtsstaatsprinzipien basierende Politik der Wettbewerbsverzerrung in der Demokratie vielfältigen Selbstkorrekturen und bleibt in ihrem Ausmaß weitaus weniger bedeutend, als es der skeptischen Sicht Olsons und wohl der meisten Vertreter eines ökonomischen Liberalismus entspricht. In einem größeren historischen Betrachtungshorizont zeichnen sich funktionierende Demokratien durch einen vergleichsweise hohen Offenheitsgrad ihrer Märkte und vor allem durch eine Vielfalt an gesellschaftlichen Kooperationsbeziehungen und damit an Tiefe der Arbeitsteilung aus.

„,[...] the basic insight reveals the existence of a double balance: open access and entry to organizations in the economy support open access in politics, and open access and entry in politics support open access in the economy. Open access in the economy generates a large and varied set of organizations that are the primary agents in the process of creative destruction. This forms the basis for the civil society, with many groups becoming politically active when their interests are threatened" (North et al. 2009: 2425).

Dies deutet darauf hin, dass sich eine (im substantiellen, nicht prozeduralen Sinne) liberale Wirtschaftspolitik inkremental, d. h. als Folge von Versuch Irrtum, durchsetzt, ohne dass Demokratien ordnungspolitische Regeln für ihre Wirtschaftspolitik formell eingerichtet hätten.

Eine explizite Festlegung auf ordnungspolitische Regeln ist in Demokratien auch kaum zu erwarten. Im demokratischen Parteienspektrum spielen Positionen des ökonomischen Liberalismus zumeist eine untergeordnete Rolle, wohingegen sich Politiker und Parteien gerade mit Verletzungen liberaler Prinzipien profilieren können. Die empirischen Befunde von North, Wallis und Weingast legen jedoch nahe, dass demokratische Wirtschaftspolitik in weit geringerem Maße die allgemeine wirtschaftliche 
Freiheit beschränkt, als es die politische Rhetorik im Parteienwettbewerbs vermuten lässt. Olsons Theorie der Verteilungskoalitionen scheint eine besondere historische Phase exzessiver Umverteilungs- und protektionistischer Regulierungspolitik, welche für die westlichen Wohlfahrtsstaaten der 1970er Jahre charakteristisch war, in unzulässiger Weise zu generalisieren. Für eine allgemeine politische Ökonomie demokratischer Wirtschaftspolitik wäre auch die spätere Liberalisierungsphase der Wirtschaftspolitik in den westlichen Ländern einzubeziehen; dies legen jedenfalls die Befunde eines Zuwachses an wirtschaftlicher Freiheit seit den 1980er Jahren nahe (Gwartney/Lawson 2004: 17-19).

Bemerkenswerterweise hat die anti-marktwirtschaftliche, z. T. offen auf Systemüberwindung zielende demokratische Wirtschaftspolitik der 1970er Jahre ihre Legitimation nicht wegen ihrer Verletzung ordnungspolitischer Prinzipien eingebüßt, welche im Bewusstsein der Bürger verankert wären, sondern durch die enttäuschenden Resultate: Der Anstieg der Arbeitslosigkeit, eine hohe Geldentwertungsrate, wachsende Staatsschulden, nachlassende Wachstumsraten und dadurch verringerte Lohnzuwächse erwiesen sich in dieser Zeit als die Geiseln demokratischer Wirtschaftspolitik. Die anschließende liberale Wende, damals als Thatcherismus, Reagonomics oder Neoliberalismus bezeichnet, lässt sich nicht als Wiederbelebung einer individualethisch gestützten Ordnungsethik deuten, welche Wähler und Interessengruppen zu einer Einsicht in die kollektive Irrationalität ihrer (individuell rationalen) Interessenwahrnehmung bewogen hätte. Die Lernprozesse müssen wohl nüchterner als ein wirtschaftspolitischer Trial-and-Error-Prozess interpretiert werden, weil die bis dahin dominierende keynesianische Doktrin in der Wirtschaftspolitik ihr Renommee aufgezehrt hatte, ohne dass dies von einer tieferen und stabilen Einsicht der demokratischen Öffentlichkeit in die Notwendigkeit liberaler Ordnungspolitik begleitet wäre. Darum lässt sich die Abkehr vom Keynesianismus der 1970er Jahre keineswegs als Rückbesinnung auf ordnungspolitische Prinzipien im Sinne eines substantiellen ökonomischen Liberalismus deuten. Denn trotz einer Politik der Wettbewerbsöffnung, Entstaatlichung und Rücknahme der Spitzensteuersätze rüttelte die Reformpolitik keineswegs generell am Wohlfahrts- und Umverteilungsstaat. Einer allgemeinen Wahrnehmung über die neo-liberale Wirtschaftspolitik zum Trotz, erfuhr der Wohlfahrts- und Regulierungsstaat sogar noch einen weiteren Ausbau. ${ }^{9}$ Er blieb als legitimationsstiftendes Fundament der westlichen Wirtschaftsordnung unangetastet. Die politischen Reformen zielten vor allem auf die Verbesserung der Anreize von Unterstützungsleistungen im Falle von Bedürftigkeit, nicht jedoch auf eine allgemeine Rückführung der Umverteilung.

Ordnungspolitische Prinzipien stehen darum stets in einem Spannungsverhältnis zu den Präferenzen der Bürger über zustimmungsfähige Regeln der Wirtschaftspolitik („Verfassungspräferenzen“). Leben die Bürger in politischen Verhältnissen, in denen sie ihre politischen Meinungen frei äußern können und ihre politischen Agenten gege-

Dies zeigte sich u.a. an den Hartz-Reformen und der Einführung des Arbeitslosengeldes II, welche den Kreis der Anspruchsberechtigten unbeabsichtigt ausweitete und einen deutlich höheren Mittelbedarf erforderte, als die damalige Bundesregierung erwartete. Danach ergänzten die nachfolgenden Regierungen den Katalog wohlfahrtsstaatlicher Leistungen durch die Einführung des Elterngeldes nochmals bzw. vermieden Reformen bei der Pflegeversicherung 
benenfalls abwählen können, muss jeder daraus resultierende Konsens über die Prinzipien der Wirtschafts- und Sozialpolitik als legitim gelten. In dieser Weise hat Buchanan auf vertragstheoretischer Grundlage den ökonomischen Liberalismus neu zu begründen versucht..$^{10}$ Legitimation stiftet ein Konsens allerdings unabhängig davon, auf welche Wirtschaftspolitik sich die Gesellschaftsmitglieder einigen. Auch eine im Konsens betriebene Umverteilungspolitik wäre legitim, selbst wenn es dabei zu allgemeinen Wohlstandseinbußen käme, um deren Vermeidung es den Vertretern des ordnungspolitischen Denkens im Wesentlichen geht. In der Demokratie, welche die Bürger zu Koautoren ihrer Politik macht, können legitime wirtschaftspolitische Regeln nur noch prozedural und nicht substantiell bestimmt werden. Eine Abwahl oder Überwindung der marktwirtschaftlichen Ordnung ist durch eine Rückbindung der Wirtschaftspolitik an die Interessen der Bürger allerdings nicht zu erwarten; die Ausflüge von Demokratien in sozialistische und andere anti-liberale Ordnungsexperimente waren im Rückblick nur von kurzer Dauer. Dennoch ermangelt es liberalen Ordnungsprinzipien, wie sie Hayek in konsequenter Weise einfordert, an allgemeiner $\mathrm{Zu}$ stimmungsfähigkeit. Offenkundig suchen Demokratien laufend nach Wegen, konstitutionelle Prinzipien der Marktwirtschaft (Schutz der Eigentumsrechte, der Vertragsfreiheit und allgemein der Nicht-Diskriminierung) mit dazu konfligierenden egalitaristischen politischen Zielen zu verbinden. Dieser „demokratische Grundwiderspruch“, Wohlstand und Sicherheit zugleich realisieren zu wollen, lässt sich von einem ordnungsökonomischen Standpunkt offenlegen und kritisieren. Im demokratischen Diskurs können solche Argumente jedoch keine „höhere“ Legitimation beanspruchen oder im Namen der Individuen auftreten. Dabei hilft es wenig, dass ordnungsökon omische Positionen einen ethischen Anspruch erheben und diesen zu begründen vermögen. Denn dies tun rivalisierende „umfassende Lehren“ (Rawls 1993), die auf Regulierung, Umverteilung und die Einhegung des Wettbewerbs zielen, in gleicher Weise. Es kommt allein darauf an, dass liberale Positionen die Mehrheit zu überzeugen vermögen, wovon in Anbetracht einer populären Kritik am Neoliberalismus oder der Globalisierung nicht auszugehen ist. Nicht ohne Grund finden liberale Argumente erst dann Gehör, nachdem die Bürger ihre Erfahrungen mit antiliberalen Politiken gemacht haben. Auch dann bleibt das ordnungspolitische Fundament demokratischer Wirtschaftspolitik brüchig.

\section{Ordnungspolitik als epistemisches Problem}

Ein zentrales Problem für die Durchsetzungsfähigkeit liberaler Argumente auf dem Marktplatz der politischen Ideen stellt der hohe Grad an Theoriegebundenheit dar, der ein Spannungsverhältnis zum individualistischen Anspruch des Liberalismus entstehen lässt. ${ }^{11}$ Der (substantielle) ökonomische Liberalismus nimmt realistischer Weise an, dass die Bürger ein Interesse an der fortgesetzten Steigerung ihres Wohlstandes

10 Zur Unterscheidung zwischen substantiellem und prozeduralem ökonomischem Liberalismus vgl. Wegner (2009) und (2011).

11 Vanberg/Buchanan (1989) erkennen dieses Problem, thematisieren jedoch nicht die Konsequenzen für den vertragstheoretischen Ansatz, der eine liberale Wirtschaftspolitik zugleich konstitutionalisieren und legitimieren will. 
haben werden, unabhängig davon, welches Wohlstandsniveau sie bereits erreicht haben. Aus diesem unterstellten kollektiven Wohlstandsinteresse schöpft der Liberalismus seine Legitimation, und zwar aus der allgemeinen wirtschaftshistorischen Beobachtung heraus, dass die Menschen ihre materiellen Bedingungen stets zu verbessern suchen, wenn sie hierzu auch die Freiheit besitzen. Daraus schöpft der Liberalismus zugleich seinen kritischen Gehalt insbesondere gegenüber Regierungsformen, in denen die Bürger die Regierenden nicht zu kontrollieren vermögen. Bis in die jüngste Gegenwart spricht die Erfahrung mit solchen Regimen dafür, dass autokratische oder oligarchische Machthaber das Gewaltmonopol des Staates dazu ausnutzen, nicht nur ihre persönliche Macht zu steigern, sondern auch ihren materiellen Wohlstand auf Kosten des gesellschaftlichen Wohlstands. In diesen Gesellschaften existieren zwar Märkte, aber kein freier Wettbewerb, weshalb breite Schichten in der Gesellschaft in Armut verharren.

Im Gegensatz dazu erscheinen Demokratien aufgrund der wettbewerblichen, temporären Zuweisung von politischer Macht geradezu prädestiniert, den allgemeinen Wohlstand zu befördern. Es entspricht der Erfahrung, dass Wähler ihre gewählte Regierung vor allem an der Entwicklung ihres persönlichen Wohlstandes messen. Das Realeinkommen Aller lässt sich aber langfristig nur unter einer liberalen, marktöffnenden Wirtschaftspolitik steigern, was einen permanenten Strukturwandel ermöglicht und akkommodierende politische Maßnahmen erforderlich machen kann. Anders als in einer Autokratie kann eine demokratische, von Abwahl bedrohte Regierung, nur auf die fortgesetzte Steigerung der Produktivität setzen, wohingegen einer Politik der Umverteilung Grenzen gesetzt sind. Eine dauerhafte wirtschaftliche Benachteiligung von großen Gruppen lässt sich in der Demokratie nur begrenzt durchhalten; ebensowenig kann sich die Mehrheit dauerhaft auf Kosten einer Minderheit bereichern. Nur in einer Autokratie verfügen die Machthaber über zahlreiche Möglichkeiten der Bereicherung. Sofern sie nicht unmittelbar aufstrebende Bürger enteignen, besteht die gängige Bereicherungsmethode in der Zuteilung von Marktzugangsrechten (vorrangig an die Machthaber und ihre Clans) oder im Verkauf solcher Rechte, während der Rest der Bevölkerung in seinen wirtschaftlichen Rechten, insbesondere Marktzugangsrechten, eingeschränkt bleibt. ${ }^{12}$ Dem gesellschaftlichen Wunsch nach einer allgemeinen Wohlstandserhöhung kann im Zweifel mit politischer Repression begegnet werden. Im Unterschied dazu kann eine Demokratie den Marktzugang nur begrenzt privilegieren und größere Bevölkerungsgruppen von der Teilnahme am Wettbewerb ausschlieBen. Ebenso sind die Möglichkeit einer Befriedigung der materiellen Wünsche der Mehrheit durch Enteignung oder übermäßige Besteuerung einer Minderheit begrenzt, anders als es manche Zerrbilder der Demokratie von einer Herrschaft über die Minderheit vermuten lassen. Einer demokratischen Regierung verbleiben nur wenig andere Möglichkeiten, den allgemeinen Wohlstand zu erhöhen, als einen Prozess der allgemeinen Produktivitätserhöhung in Gang zu setzen. Dies aber verlangt objektiv eine liberale Wirtschaftspolitik, also insbesondere die Verwirklichung aller ökonomischen die sich - entgegen mancher Hoffnung - zu Autokratien zurückgebildet haben, findet sich bei Timm (2010). 
Grundfreiheiten, welche in undemokratischen Gesellschaften, falls überhaupt, nur unvollständig verwirklicht sind (Vertragsfreiheit, Freihandel, Niederlassungsfreiheit, Berufsfreiheit, Schutz der Eigentumsrechte).

Damit ist jedoch nur die objektive Ausgangslage demokratischer Wirtschaftspolitik beschrieben, ohne dass man daraus vorherrschende politische Präferenzen deduzieren könnte. Der substantielle ökonomische Liberalismus ist in epistemischer Hinsicht zu voraussetzungsvoll, als dass der Souverän der Demokratie entsprechende Präferenzen für eine liberale Wirtschaftspolitik ausbilden würde. Dafür müssten den Bürgern die institutionellen Bedingungen für die Entstehung materiellen Wohlstandes zunächst einmal einleuchten. Andernfalls werden sie wirtschaftspolitische Ziele präferieren, die mit dem Wohlstandsziel konkurrieren oder dieses sogar gefährden. Davon ist jedoch im allgemeinen Fall auszugehen. Eine Mehrheit für eine anti-liberale Wirtschaftspolitik braucht noch nicht einmal in der Wahrnehmung eigennütziger Gruppeninteressen begründet liegen oder in der allgemeinen Ablehnung auf Seiten der Bürger, politische Ziele vom Standpunkt einer kollektiven Rationalität aus beurteilen. Es ist durchaus denkbar und auch empirisch bedeutsam, dass die Mehrheit der Bürger eine liberale Wirtschaftspolitik aus Gründen ablehnt, die ihnen als Wahrnehmung eines Allgemeinwohls erscheinen. Das ist zum Beispiel dann der Fall, wenn universalistische Regeln, welche der klassische Liberalismus fordert, im öffentlichen Diskurs keine Unterstützung finden, wohl aber die Begünstigung von Gruppen oder Industriesektoren zur Beförderung eines ausgerufenen Gemeinwohlziels. Die Mehrheit mag verkennen, dass sie für eine Begünstigung zahlt oder aber ihre Zahlung akzeptieren. Vor allem aber dürfte sich der Öffentlichkeit nicht unmittelbar erschließen, dass eine Beschränkung der allgemeinen wirtschaftlichen Freiheit, abhängig vom Ausmaß, mit einem Verlust an allgemeinem gesellschaftlichem Wohlstand zu bezahlen ist. Dieser Zusammenhang erschließt sich nur vom Standpunkt einer (institutionen-) ökonomischen Theorie.

Es ist demzufolge nicht damit getan, dass die Gesellschaftsmitglieder ihre besonderen Handlungsinteressen zurückstellen und einen kollektiven Interessenstandpunkt einnehmen, damit sie eine liberale Ordnungspolitik unterstützen. Dazu hätten sie sich eine zentrale Erkenntnis des ökonomischen Liberalismus zu eigen zu machen, der eine evolutorische Markttheorie als theoretischen Kern enthält: dass offener Wettbewerb, permanenter Strukturwandel und Einkommensungewissheit den Preis für die Steigerung des allgemeinen Wohlstands einer Gesellschaft bilden. Ein solcher Kausalzusammenhang ist keineswegs unmittelbar evident, als dass er in die politischen Präferenzen der Bürger als Wohlstandsinteressenten einfließen würde, sobald diese nur von ihren situativen Eigeninteressen abstrahierten. In diesem Sinne hatten Vanberg und Buchanan (1989) analysiert, dass rationale Individuen zur Konstituierung einer liberalen Ordnung einen kollektiven Interessenstandpunkt einzunehmen hätten, um situative Handlungsinteressen in Verfassungsinteressen oder Ordnungsinteressen zu tran sformieren. Ordnungsinteressen lassen sich dabei nicht unmittelbar aus individuellen Eigeninteressen deduzieren, werden aber durch letztere gestützt. Es ist evident, dass Individuen eine solche Transformation ihrer situativen Handlungsinteressen in ein konsensuelles Ordnungsinteresse erst vornehmen können, wenn sie um die wohlstandssteigernde Wirkung der Ordnungsregeln wissen. Solche epistemischen Annah- 
men lassen sich jedoch den Bürgern nicht einfach extern zuschreiben, um die Chancen für einen Ordnungskonsens nachzuweisen. Bereits die Ökonomik wartet mit konkurrierenden Theorien über die Entstehung von Wohlfahrt auf und hat die Bedeutung des rechtlichen und politischen Ordnungsrahmens für die Marktallokation zeitweilig völlig aus dem Blick verloren. Umso weniger wird man von Bürgern erwarten dürfen, dass ihnen die Bedeutung marktwirtschaftlicher Ordnungspolitik für den gesellschaftlichen Wohlstand bewusst wäre.

Durch die Verdrängung von (Rechts-) Institutionen aus der ökonomischen Theorie wurde ein möglicher Zielkonflikt zwischen wohlfahrtsschaffender Freiheit und Sicherheit als Ziel demokratischer Politik lange Zeit gar nicht thematisierbar. Das gilt sowohl für die allgemeine Gleichgewichtstheorie und den 2. Hauptsatz der Wohlfahrtsökonomik als auch für die Makroökonomik der 1970er Jahre. Die Auffassung, dass Wohlstand und allgemeine Einkommenssicherheit zugleich zu haben sind, entspricht nicht nur einer auch gegenwärtig vorherrschenden gesellschaftlichen Überzeugung; sie fand (und findet) auch von Seiten der ökonomischen Theorie Unterstützung. Erst Hayek hatte den liberalen Gedanken erneuert, dass freiheitssichernde Institutionen des Rechtsstaates einerseits Wohlstand ermöglichen, andererseits aber einen politischen Kontrollverlust für gesellschaftliche Entwicklungen zur Folge haben (Hayek 1971).

Das bedeutet nicht notwendigerweise, dass zwischen Einkommenssicherheit und Wohlstand eine strikte Antinomie vorläge, wie sie Hayek in seiner fundamentalen Kritik an der sozialen Gerechtigkeit behauptet und die ihn zum Verfechter einer konsequent liberalen Wirtschaftspolitik ohne nennenswerte Umverteilungselemente werden lässt. Es liegt wohl eher ein Trade-off vor, den eine demokratische Gesellschaft auch anders entscheiden kann, als es Hayek für legitim erachtet. In jedem Fall ist zu entscheiden, wie attraktiv die Wahrnehmung von politisch gewährten Transfereinkommen einerseits und wie unattraktiv die risikobehaftete Suche nach Unternehmergewinnen andererseits ausfallen und institutionell gestaltet sein soll. Die Schärfe des Zielkonflikts hängt allerdings auch von kontingenten Wachstumsbedingungen einer Volkswirtschaft ab; eine günstige Wachstumsphase kann den negativen Einfluss der Wirtschaftspolitik phasenweise überkompensieren, so dass diese zeitweilig ungewöhnlich große Spielräume für die Umverteilung von Einkommen und die Regulierung wirtschaftlicher Aktivitäten genießen mag. Dies ist wohl in den meisten westlichen Ländern in der Nachkriegszeit der Fall gewesen, als viele außerökonomische Faktoren für ein günstiges Wachstumsumfeld beitrugen und ein Verständnis für die Bedeutung liberaler Ordnungspolitik verdrängten. ${ }^{13}$ Erst mit dem Auslaufen des Nachkriegsbooms wurde jedoch nach und nach offensichtlich, dass wirtschaftliche Dynamik ordnungspolitischer Voraussetzungen bedarf, welche die Spielräume eines wirtschaftspolitischen Dezisionismus durchaus begrenzen können. Diese Erkenntnis setzte sich allerdings nur zögerlich durch und kann keineswegs zum festen Erfahrungs-

13 Zu diesen Faktoren gehörte ein hoher Nachholbedarf im Konsum, vielfältige unternehmerische Chancen durch technologische Erfindungen der Vorkriegs- und Kriegszeit (Kondratief-Zyklus) und nicht zuletzt eine geringe Standortkonkurrenz der Marktwirtschaften in der Weltwirtschaft, unter anderem auf Grund des Umstandes, dass viele Regierungen in der Welt nichtmarktwirtschaftliche Ordnungsexperimente ausprobierten. 
schatz westlicher Demokratien gezählt werden. So scheint die Finanzmarktkrise zeitweilig die Auffassung genährt zu haben, dass wirtschaftliche Freiheit generell eine Gefahr für das Allgemeinwohl darstellt und Regulierungsdefizite in der Finanzbranche pars pro toto gelten. In diesem Sinne haben Habermas und Ulrich Beck auf dem Höhepunkt der Finanzmarktkrise eine fundamentale Krise des „Neoliberalismus“ ausgerufen und ein Primat der Politik gefordert, das in der Konsequenz nichts anderes meinen kann als die generelle Einhegung der wirtschaftlichen Freiheit. ${ }^{14}$ Vor dieser Konsequenz schrecken beide Autoren allerdings bemerkenswerter Weise zurück, wie überhaupt die aufflackernde öffentliche Kapitalismuskritik seltsam inkonsequent und rückwärtsgewandt ausfiel. Dennoch wiesen die brüchigen Grundlagen demokratischer Ordnungspolitik darauf hin: Demokratische Gesellschaften besitzen nur ein unzureichendes Verständnis von den institutionellen Voraussetzungen ihres materiellen Wohlstands, weshalb eine liberale Ordnungspolitik keinen expliziten gesellschaftlichen Konsens findet. Das zeigt sich immer dann, wenn politische Akteure hochgespannte Gemeinwohlerwartungen an ökonomische Entwicklungsprozesse richten und bei Nichterfüllung ein Marktversagen ausrufen, womit sie ein Versagen der marktwirtschaftlichen Ordnung meinen und nicht den ökonomischen Theoriebegriff des Marktversagens. Die Kapitalismuskritik nährt das planwirtschaftliche Sentiment, dass allgemeiner Wohlstand qua politischer Dezision herstellbar sei und nicht die Resultante von spontanen Marktprozessen darstellt, welche die Politik bestenfalls indirekt fördern kann. Auf diese Weise entsteht leicht eine öffentliche Debattenkonstellation, in der sich anti-liberale Interventionisten zu Anwälten von Gemeinwohlzielen machen (,,jeder muss von seiner Arbeit leben können“), wogegen die Vertreter liberaler Ordnungspolitik längere Argumentationsketten entfalten müssen, die den Kontakt mit den Arenen demokratischer Medienkultur nicht immer gut vertragen.

Wohlgemerkt hat auch die ökonomische Theorie den kausalen Zusammenhang zwischen freiheitssichernden Institutionen und wirtschaftlichem Wohlstand erst seit einiger Zeit wieder aufgegriffen, obwohl es sich um einen Gedanken handelt, der sich durch das gesamte „Wealth of Nations" bei Adam Smith zieht. In der modernen Ökonomik ist das politische, rechtliche und kulturelle Bezugssystem eines prosperierenden Kapitalismus verloren gegangen. Es ist noch nicht lange her, dass die Relevanz von Unternehmertum und Innovationswettbewerb für die wirtschaftliche Dynamik von Volkswirtschaften ausgeblendet blieb und wirtschaftlicher Wohlstand als deterministische Resultante makroökonomischer Steuerungsinstrumente gedeutet wurde. Eine ökonomisch bedeutsame Rolle für den Freiheitsbegriff war in diesen Makromodellen ebenso wenig vorgesehen wie für den Innovationswettbewerb als Quelle gesellschaftlichen Wohlstandes. Erst mit Nelson und Winter (1982) wandte sich die ökonomische Theorie einer evolutorischen Betrachtungsweise von wirtschaftlicher Dynamik zu. Das zeigte sich vor allem darin, dass sie dem Innovationsbegriff, der zuvor bestenfalls einen Appendix der ökonomischen Theorie darstellte, nunmehr paradigmatische Bedeutung beimaß. Für diese grundlegende theoretische Umorientierung dürften die bereits angesprochenen wirtschaftspolitischen Erfahrungen maßgeblich gewesen sein. Nach dem Auslaufen des Nachkriegsbooms setzte eine hartnäckige

14 Vgl. Habermas (2008) und Beck (2008). 
Stagnationskrise ein, die durch ausufernde Staatsverschuldung, beschleunigte Inflation und ungewohnt hohe Arbeitslosigkeit geprägt war. Der Versuch einer Einhegung der Krise durch geld- und fiskalpolitische Maßnahmen zeitigte keine nachhaltigen Erfolge mehr. Vor diesem Hintergrund setzte in der ökonomischen The orie eine Neuorientierung bei der Suche nach günstigen Wachstumsbedingungen ein, die als grundlegender Paradigmenwechsel wahrgenommen wurde. Dieser fand zunächst in der ökonomischen Theorie statt, erfasste dann aber auch die demokratische Öffentlichkeit. Man kann diese Erfahrung aber auch als Hinweis dafür nehmen, welche Rationalitätserwartungen an den Souverän der Demokratie realistischerweise gestellt werden dürfen. Es ist nicht davon auszugehen, dass die Bürger als Ko-Autoren der Politik die Funktionsweise einer marktwirtschaftlichen Ordnung hinreichend verstehen, um ihr Wohlstandsinteresse gegen dazu konkurrierende Politikziele rational abwägen zu können. Entsprechend können ihre politischen Präferenzen in Widerspruch zu ihren eigenen geäußerten Einkommensinteressen geraten. Damit aber entfällt eine Voraussetzung für die Ausbildung konsistenter Präferenzen. Präferieren die Bürger wachsenden Wohlstand, verweigern als Wähler aber einer liberalen Wirtschaftspolitik ihre Unterstützung, so vermögen sie ihre politischen Agenten nicht auf der Basis informierter Präferenzen zu kontrollieren. Epistemische Gründe dürften maßgeblich dafür sein, dass Bürger ordnungspolitische Handlungen ablehnen, deren Wohlstandskonsequenzen sie jedoch wünschen. So entspricht es der Erfahrung, dass Bürger in einer wirtschaftspolitischen Reformphase einer Politik der Wettbewerbsöffnung häufig skeptisch bis ablehnend gegenüber stehen, selbst wenn sie einer Gruppe angehören, die von der Wettbewerbsöffnung unmittelbar gar nicht betroffen ist. In solchen Fällen kann man die Ablehnung einer liberalen Wirtschaftspolitik nicht auf den Antagonismus zwischen eigenem (Gruppen-) Wohl und öffentlichem Interesse verkürzen, obwohl Eigeninteressen bei der Bewertung von politischen Handlungen immer im Spiel sind. Darüber hinaus gehen Liberalisierungsphasen in der Regel mit einer „Anfangsverschlechterung“ (,J-Curve") einher, deren Dauer ungewiss ist und auch von wirtschaftspolitisch unbeeinflussbaren exogenen Größen abhängt. In solchen Fällen kann sich eine Zurückweisung liberaler Wirtschaftspolitik sogar kurzfristig auf empirische Evidenz stützen. ${ }^{15}$ In dieser Phase handeln sich Anhänger einer liberalen Wirtschaftspolitik zumeist einen Ideologievorwurf ein und müssen nicht selten gegen eine gesellschaftliche Mehrheitsmeinung antreten. Da die Richtung des initiierten Strukturwandels selbst ein nicht-antizipierbarer Prozess darstellt und sich politischen Bemühungen um Beschleunigung häufig wiedersetzt, erscheint die öffentliche Unterstützung für eine liberale Ordnungspolitik höchst voraussetzungsvoll.

Rekonstruktionen des klassischen ökonomischen Liberalismus durch Buchanan oder Hayek schenken solchen epistemischen Voraussetzungen einer Regelbindung der Wirtschaftspolitik nur geringe Aufmerksamkeit. ${ }^{16}$ Liberale Ordnungspolitik erscheint

15 Ein drastisches Beispiel für die Wahrnehmung einer gescheiterten Liberalisierung stellte die Wirtschaftspolitik Thatchers in Großbritannien dar. Ebenso ist die Transformationspolitik von Balcerowicz in Polen zu Beginn der 1990er Jahre zu nennen, die aufgrund der drastischen Strukturwirkungen schon kurz nach ihrem Beginn als gescheitert galt. In beiden genannten Fällen konnten erst Nachfolgeregierungen die Früchte der Reformen ernten und für sich reklamieren.

16 Vgl insbesondere Brennen/Buchanan (1985) oder Hayek (1991).

zfwu 12/2 (2011), 250-268 
als eine allgemein zugängliche politische Idee, sobald Individuen nur ihr Gruppeninteresse, das sie durch politisch zu gewährende Sonderverteile zu bedienen trachten, zurückstellen. Eine zumeist implizite Annahme besteht darin, dass die Selbstbeschränkung von Gruppeninteressen langfristig eine Kompensation durch den Gewinn an allgemeiner wirtschaftlicher Dynamik ermöglicht. Die dadurch mögliche allgemeine Einkommenssteigerung könnte nach vollzogenem Strukturwandel alle Gruppen besser stellen. Damit besitzt eine liberale Ordnungspolitik eine ethische Grundlage, in der Algemeinwohl und individuelles Interesse nicht in einem Antagonismus stehen. Bei aller Unterschiedlichkeit der Positionen von Eucken über Hayek bis Buchanan gibt es eine Übereinstimmung dahingehend, dass liberale Wirtschaftspolitik lediglich die Einhaltung allgemeiner und damit gerechter Regeln voraussetzt und die politische Sphäre von der Verfolgung individueller und Gruppeninteressen freizuhalten ist. Der auferlegte Interessenverzicht kann die Hinnahme temporärer Einkommenseinbußen als Folge des Strukturwandels verlangen, ebenso die Akzeptanz eines ungleichen Einkommensanstiegs im wirtschaftlichen Aufschwung. Damit lässt sich die Ethik der Ordnungspolitik mit der ansonsten geläufigen Rationalitätsannahme in der Ökonomik in Einklang bringen, ohne dass sie sich allerdings von letzterer bruchlos ableiten ließe, wie Buchanan in einem groß angelegten Entwurf zu zeigen gehofft hatte. ${ }^{17}$

Aus dieser Sicht sollte es Bürgem evident erscheinen, auf die politische Verfolgung von Sonderinteressen zu verzichten und entsprechende konstitutionelle Regeln zu befürworten, welche in diesem Sinne die Handlungsspielräume politischer Akteure begrenzen. Das „öffentliche Interesse“ lässt sich durch die negativen Ordnungsfolgen eines Gruppenegoismus begründen und ist damit in normativer und kognitiver Hinsicht voraussetzungsarm. Die Bürger hätten sich lediglich klar zu machen, dass sie ein mögliches Gefangenendilemma überwinden müssen, um eine liberale Ordnungspolitik befürworten zu können. Dies verlangt nur die Einsicht, dass eine um sich greifende Wirtschaftspolitik der Regulierung, Umverteilung und Wettbewerbsbeschränkung den Wohlstand gefährdet, gesetzt dass alle Gruppen in dieser Weise ihre Sonderinteressen durchzusetzen versuchen. Eine solche kognitive Einsicht wird den Bürgern als möglich unterstellt.

Das Ordnungsproblem, eine liberale Wirtschaftspolitik durch Regeln zu verankern, ist damit jedoch nur teilweise gelöst. Denn auch die Existenz eines Gefangenendilemmas infolge einseitiger Privilegiensuche erschließt sich den Individuen keineswegs unmittelbar. Die Ordnungsfolgen könnten aus Sicht einer Gruppe immer noch vernachlässigbar gering oder langfristiger Natur sein, so dass die Verfolgung von Eigeninteressen durch politisch gewährte Vergünstigung eine attraktive Option darstellen mag. ${ }^{18}$ Insofern kann eine liberale Ordnungspolitik nicht auf ein individualethisches Fundament

17 Vgl. Buchanan (1984); für eine scharfsinnige Analyse s. Petersen (1996).

18 Eine solche Privilegiensuche mag den Individuen noch nicht einmal als Regelverletzung erscheinen, sondern als berechtigte Förderung eines Allgemeinwohlziels. So dürften etwa die Unternehmen und Beschäftigten der hochsubventionierten Solarindustrie oder Betreiber von Windkraftanlagen denken, die sich keineswegs als Subventionsempfänger empfinden dürften sondern eher als Protagonisten einer ,nachhaltigen“ Umweltpolitik. 
verzichten. ${ }^{19}$ Aber auch wenn es daran nicht mangeln sollte und Bürger bei der Abwägung ihrer (kurzfristigen) Eigeninteressen und dem allgemeinen Interesse einer freiheitssichernden Ordnungspolitik den Vorzug zu geben bereit wären: Begründungen einer liberalen Wirtschaftspolitik müssen stets voraussetzen, dass die Bürger die Ordnungsfolgen einer nicht-liberalen Wirtschaftspolitik grundsätzlich zu erkennen vermögen. Entfällt diese Voraussetzung, scheiden die Individuen als Initiatoren liberaler Ordnungspolitik aus. Diese könnte nur noch auf einem liberalen Paternalismus basieren, im Vertrauen darauf, dass die Bürger ihre Zustimmung ex post geben, weil sie die positiven Wohlstandsfolgen überzeugen. Ein liberaler Paternalismus wiederum liefe nach der hier vertretenen Auffassung auf einen liberalen Selbstwiderspruch hinaus: denn eine liberale Ordnung impliziert in erster Linie, dass sich die Bürger ihre Gesetze und Regeln in Freiheit selbst geben. ${ }^{20}$

\section{Zusammenfassung und Ausblick: Demokratische Ordnungspolitik als Trial und Error}

Aufgrund der epistemischen Voraussetzungen einer liberalen Wirtschaftspolitik reichen die Wohlstandsinteressen der Bürger allein nicht hin, um Bürger ,wie sie sind“ (Buchanan) als Rechtfertigungsgrund für liberale Ordnungsregeln anzurufen. Demokratische Ordnungspolitik beinhaltet das, was der Souverän der Demokratie nach seinem Wissens- und Erfahrungsstand für regelungsbedürftig hält. Dies kann durchaus eine absichtsvolle Zurückdrängung des Wettbewerbsprinzips bedeuten, die von einer breiten Mehrheit getragen wird, obwohl daraus Wohlstandseinbußen resultieren. Es ist keine triviale Frage, warum empirische Bürger zentrale Elemente einer liberalen Wirtschaftspolitik ablehnen, obwohl sie damit ihren geäußerten Wohlstandsinteressen erkennbar schaden. ${ }^{21}$ Dies ist umso bemerkenswerter, als die Bürger damit keineswegs immer ihre situativen Eigeninteressen im Blick haben, wie die unpopuläre Liberalisierungs- und Deregulierungspolitik in den 1990er Jahren belegt. Als durch den wachsenden Einfluss der europäischen Wirtschaftsverfassung jahrzehntelang geschützte Staatsmonopole wie Telekommunikation, Gas, Strom, Bahn und Post in den Wettbewerb (teilweise) eingegliedert wurden, überwog bei den Wählern durchaus eine Skepsis gegenüber dieser Liberalisierung - trotz des Umstandes, dass eine Mehrheit unter den Wählern keine Beschäftigteninteressen zu verteidigen hatte. Ihre Zustimmungsfähigkeit gewann die durch europäisches Recht erzwungene Wettbewerbsöffnung erst im Nachhinein, als die Bürger von der Intensivierung des Wettbewerbs profitierten.

Ein stabiler ordnungspolitischer Konsens zugunsten einer liberalen Wirtschaftspolitik lässt sich aufgrund solcher positiven Erfahrungen aber nicht ableiten. Auch gegenwärtig nutzen Politiker populäre Vorbehalte gegen den Wettbewerb aus, um etwa die Monopolstellung der Bundesbahn erfolgreich zu verteidigen. Nach der Finanzmarkt-

19 Vgl. hierzu Goldschmidt (2002), der in seiner Rekonstruktion des ORDO-Liberalismus diese individualethische Seite zu Recht betont und sich gegen Versuche wendet, diese durch eine Ordnungsethik restlos aufzulösen, wofür die Homann-Schule zumindest in ihrer Darstellungsform plädiert.

20 Im Gegensatz dazu vgl. Sunstein/Thaler (2003); für eine Kritik s. Sugden (2008)

21 Für eine Ausarbeitung dieser Thematik vgl. Wegner (2011). 
krise haben diese anti-liberalen Vorbehalte neuen Auftrieb erhalten, weil es politische Akteure durchaus erfolgreich verstanden haben, eine Deregulierungspolitik generell als Gefahr für das Allgemeinwohl darzustellen. Unter diesen Umständen muss eine Politik der Marktöffnung gegen eine Mehrheitsmeinung betrieben werden, was in der Vergangenheit vor allem durch Rechtsprechung oder Entscheidungen der Europäischen Kommission geschehen ist, deren demokratische Legitimation zumindest von einem liberalen Standpunkt kritisch hinterfragt werden darf. Denn es sind vor allem liberale Vertragstheoretiker wie Buchanan oder Brennan, die eine von Gerichten betriebene Wirtschafts- und Sozialpolitik kritisiert haben und sich von einem gesellschaftlichen Konsens über Regeln die Zurückschneidung des Interventions- und Regulierungsstaates versprochen hatten. Davon kann jedoch nicht ausgegangen werden, wie sich schon an der erfolgreichen Kampagne deutscher Politiker gegen die Bolkestein-Richtlinie der Europäischen Kommission ersehen ließ, welche den Wettbewerb für Dienstleistungen auf dem Europäischen Markt zum Nutzen der Verbraucher intensivieren wollte. Daraus ist zu folgern: Das Verhältnis der Bürger zu den konstitutiven Regeln einer marktwirtschaftlichen Ordnung ist ambivalent; denn das allgemeine Wohlstandsinteresse übersetzt sich nicht oder nur begrenzt in eine Präferenz für ordnungspolitische Handlungen.

Das bedeutet nicht, dass die Bürger den Weg in eine antiwettbewerbliche Wirtschaftspolitik dauerhaft mittragen würden und eine liberale Ordnungspolitik chancenlos wäre. Zwar lassen sich inzwischen neue politische Handlungsfelder ausmachen, in denen eine immer striktere Regulierungspolitik betrieben wird. Umweltpolitik, Gleichstellungspolitik, Lohnpolitik (Mindestlöhne), Verbraucherschutz und immer dichtere Corporate Governance-Regeln für Unternehmen bieten Beispiele eines neuen Interventionismus und Regulierungsstaates. Die dahinter stehenden Steuerungsabsichten erfreuen sich zwar noch breiter gesellschaftlicher Zustimmung. Diese kann allerdings erodieren, sobald der neue Interventionismus nicht nur die bürgerliche Freiheit immer stärker beschneidet, sondern auch unabweisbare Wohlstandsrisiken zeitigt. In der regulativen, auf Vorschriften und Verbote setzenden Umweltpolitik zeichnet sich bereits der Beginn eines Legitimationsdefizits ab, das sich mit dem noch zu erwartenden Kostenschub einer auch ökologisch fragwürdigen Quotenpolitik rasch beschleunigen kann. Es bleibt abzuwarten, ob die Bürger eine solche regulative und kostenintensive Umweltpolitik unbegrenzt mittragen werden oder eine Rückbesinnung zu ordnungskonformeren und damit kostengünstigeren Politikvarianten erfolgen wird.

Es sind jedoch nicht die ordnungspolitischen Überzeugungen des Souveräns der Demokratie, die ein zuverlässiges Gegengewicht zum ausufernden Interventionsstaat bilden, sondern vielmehr das kollektive Wohlstandsinteresse. In diesem Spannungsfeld bewegt sich eine demokratische Ordnungspolitik und unterscheidet sich hierin von einer an Prinzipien und Regeln orientierten, theoretisch deduzierten liberalen Ordnungspolitik. Der Unterschied macht sich vor allem an der Form fest. Demokratische Ordnungspolitik gewinnt ihre Prinzipien aus kollektiven Lernprozessen und stattet sie mit vorläufiger Geltung aus. Um Erfahrungsprozessen Raum zu verschaffen und gesetzgeberische Entscheidungen nicht zu überlasten, wird es dem demokratischen Gesetzgeber stets rational erscheinen, dem Gesetzesvollzug und damit der Verwaltung Entscheidungsspielräume zu verschaffen, die eine Korrektur aufgrund 
von Erfahrungen ermöglichen. Gerade in Rechtsräumen, die von hoher rechtsstaatlicher und öffentlicher Kontrolle geprägt sind, eröffnet sich dadurch ein Raum für Lernprozesse. Sie können genutzt werden, um gesetzgeberische Maßnahmen zu korrigieren, ohne eine politisch kostspielige Gesetzesrevision in Gang setzen zu müssen. Die formale Gesetzesrevision mag dann später zu einem politisch opportun erscheinenden Zeitpunkt erfolgen. In dieser Weise haben es die westlichen Demokratien in der jüngeren Vergangenheit immer wieder vermocht, den Katalog politischer Gestaltungsziele zu vermehren und gleichzeitig Kompromisse mit einer wohlstandssichernden Politik der Wettbewerbsöffnung zu finden. Dieser eigensinnige Charakter demokratischer Ordnungspolitik lässt zwar das Wohlstandspotential moderner Demokratien unausgeschöpft. Es ist jedoch kein anderes politisches System in Sicht, welches die wirtschaftliche Freiheit auf Dauer in höherem Maße schützt.

\section{Literaturverzeichnis}

Beck, U. (2008): „Die Finanzmarktkrise hat aus Schurken Helden gemacht“; Interview im Spiegel vom 15.10.2008.

Brennan, G./ Buchanan, J. M. (1985): The Reason of Rules. Constitutional Political Economy, (Cambridge University Press).

Goldschmidt, N. (2002): Entstehung und Vermächtnis ordoliberalen Denkens. Walter Eucken und die Notwendigkeit einer kulturellen Ökonomik, Münster: LIT-Verlag.

Gwartney, J./ Lawson, R. (Hrsg.)(2004/2005): Economic Freedom of the World, The Fraser Institute, published by The Liberal Institute of the Friedrich Naumann Foundation, Berlin: ESM.

Habermas, J. (2008): „Nach dem Bankrott, Der Privatisierungswahn ist an sein Ende gekommen. Nicht der Markt, sondern die Politik ist für das Gemeinwohl zuständig: Ein Gespräch mit dem Philosophen Jürgen Habermas über die Notwendigkeit einer internationalen Weltordnung“; in: DIE ZEIT, 6.11.2008 Nr. 46.

Hayek, F. A .v. (1991/1971): Die Verfassung der Freiheit, 3. Aufl., Tübingen: Mohr.

Hayek, F. A. v. (2007/1971): Der Weg zur Knechtschaft, München: Olzog.

Nelson, R. R./ Winter, S. (1982): An Evolutionary Theory of Economic Growth, Cambridge.

North, D. C./ Wallis, J./ Weingast, B. (2009): Violence and Social Orders. A Conceptual Framework for Interpreting Recorded Human History, Cambridge: Cambridge University Press.

Rawls, J. (1998/1993): Politischer Liberalismus, Frankfurt a. M.: Suhrkamp.

Petersen, T. (1996): Individuelle Freiheit und allgemeiner Wille. Buchanans politische Ökonomie und die politische Philosophie, Tübingen: Mohr.

Sugden, R. (2008): „Why incoherent preferences do not justify paternalism“; in: Constitutional Political Economy, Vol. 19, 226-248.

Sunstein, C./ Thaler, R. (2003): „Libertarian Paternalism“, American Economic Review, Papers and Proceedings, Vol. 93 [2], 175-179.

Timm, C. (2010): Jenseits von Demokratiehoffnung und Autoritarismusverdacht: Eine herrschaftssoziologische Analyse post-transformatorischer Regime, In: Albrecht, H./ Frankenberger, R. (Hrsg.): Autoritarism Reloaded. Neuere Ansätze und Erkenntnisse in der Autokratieforschung, Nomos, 95-122. 
Vanberg, V./ Buchanan, J. M. (1989): Interests And Theories In Constitutional Choice; in: Journal of Theoretical Politics, Vol. 1, 49-62.

Wissenschaftlicher Beirat beim Bundeswirtschaftsministerium (2004): Zur Förderung Erneuerbarer Energien, www.stoerwind.de/WissBeirat.pdf.

Wegner, G. (2008): Political Failure by Agreement. Learning Liberalism And The Welfare State, Cheltenham: Elgar.

Wegner, G. (2009): „Substantive versus Procedural Liberalism: Exploring a Dilemma of Contemporary Liberal Thought“; in: Journal of Institutional and Theoretical Economics, Vol. 165, 535-557.

Wegner, G. (2011): Ökonomischer Liberalismus als politische Theorie. Befund, Kritik, Rekonstruktion, Tübingen: Mohr. 